\title{
More than meets the eye: visual attention biases in individuals reporting chronic pain
}

\section{Samantha R Fashler Joel Katz}

Department of Psychology, York University, Toronto, ON, Canada

Correspondence: Samantha Fashler or Joel Katz

Department of Psychology,

York University, 4700 Keele Street, BSB 232, Toronto, ON M3J IP3, Canada

Tel + I 4167362100 ext 40557

Fax +I 4167365814

Email sfashler@yorku.ca or

jkatz@yorku.ca

\author{
This article was published in the following Dove Press journal: \\ Journal of Pain Research \\ 19 September 2014 \\ Number of times this article has been viewed
}

\begin{abstract}
The present study used eye-tracking technology to assess whether individuals who report chronic pain direct more attention to sensory pain-related words than do pain-free individuals. A total of 113 participants (51 with chronic pain, 62 pain-free) were recruited. Participants completed a dot-probe task, viewing neutral and sensory pain-related words while their reaction time and eye movements were recorded. Eye-tracking data were analyzed by mixeddesign analysis of variance with group (chronic pain versus pain-free) as the between-subjects factor, and word type (sensory pain versus neutral) as the within-subjects factor. Results showed a significant main effect for word type: all participants attended to pain-related words more than neutral words on several eye-tracking parameters. The group main effect was significant for number of fixations, which was greater in the chronic pain group. Finally, the group by word type interaction effect was significant for average visit duration, number of fixations, and total late-phase duration, all greater for sensory pain versus neutral words in the chronic pain group. As well, participants with chronic pain fixated significantly more frequently on pain words than did pain-free participants. In contrast, none of the effects for reaction time were significant. The results support the hypothesis that individuals with chronic pain display specific attentional biases toward pain-related stimuli and demonstrate the value of eye-tracking technology in measuring differences in visual attention variables.
\end{abstract}

Keywords: attention, chronic pain, dot-probe task, eye-tracking

\section{Introduction}

Selective attention to pain-related information is implicated as contributing to the cause and maintenance of chronic pain. ${ }^{1,2}$ This "attentional bias" involves an increase in the allocation of attentional resources to the threatening object, including the orientation, ${ }^{3}$ maintenance, ${ }^{4}$ and disengagement of attention. ${ }^{5}$ However, evidence for attentional biases toward pain-related information in individuals with chronic pain is inconsistent ${ }^{6-9}$ and may be related to the methodological limitations in its measurement.

Attentional biases have traditionally been inferred using paradigms such as the dotprobe task that use reaction time as the primary measure. The dot-probe task typically involves the simultaneous presentation of a threat word and a neutral word on either side of a computer screen. ${ }^{10}$ The two words remain on the screen for a short duration, after which a dot is presented in the location of one of the words. The participant is to indicate the side of the screen on which the dot appears as quickly and accurately as possible by pressing one of two keys. The presence of an attentional bias toward threat-related stimuli is inferred by a shorter mean reaction time to the dot when it is presented in the location of the threat word than when the dot is presented in the location of the neutral word. ${ }^{10}$ 
Despite its frequent use, the dot-probe task has limitations. Most prominently, the primary outcome measure of the task is reaction time, an indirect indicator of attention; it assumes that faster reaction times accurately reflect selective visual attention toward the threatening stimulus. A more accurate index of visual attention would be a measure of the eye movements of participants. To date, only one study that we are aware of has investigated visual attention patterns in the dot-probe task using eye-tracking technology in individuals with chronic pain. ${ }^{11}$ The authors tracked the eye movements of 24 adults with and without chronic pain who were either high or low in fear of pain during a modified dot-probe task consisting of pain-neutral, health catastrophe-neutral, and neutral-neutral word pairs. The results did not show significant differences in reaction time according to pain status, fear of pain, or word type. However, compared with individuals who did not have chronic pain, those with chronic pain had shorter first fixation durations to health catastrophe words. These results suggest that eye-tracking may be more sensitive at identifying attentional biases than the traditional reaction time measure used for the dot-probe task. ${ }^{12}$

Traditional dot-probe tasks are also limited in their ability to capture attentional biases across phases of visual attention. ${ }^{12}$ Visual attention phases represent periods of attentional processing toward a stimulus that are qualitatively and temporally distinct, including initial orientation and engagement, ${ }^{13,14}$ maintenance and disengagement, ${ }^{2,15,16}$ avoidance, ${ }^{17}$ and reengagement. ${ }^{18}$ It is possible that visual attention biases differ across visual attention phases, which have been assessed with varying exposure durations of word pairs in the classical dot-probe task. As a continuous measure of visual attention, eye-tracking permits the capture of attention at different visual attention phases within the same trial, although this has yet to be investigated.

A thorough understanding of how attentional biases relate to chronic pain must consider the biopsychosocial context of the individual. ${ }^{19}$ In particular, psychological variables are linked to the development and maintenance of chronic pain. ${ }^{19}$ Constructs related to anxiety are well-established, linking levels of pain catastrophizing, ${ }^{20}$ fear of pain, ${ }^{21}$ sensitivity to anxiety or illness, ${ }^{22}$ pain anxiety, ${ }^{23}$ state anxiety, ${ }^{24}$ symptoms of posttraumatic stress disorder, ${ }^{25}$ pain vigilance, ${ }^{26}$ and acceptance of chronic pain ${ }^{27}$ to the experience of pain. Evaluating the relationship between psychological constructs related to pain and outcome measures, such as reaction time and eye-tracking parameters, will permit a more thorough understanding of attentional biases in the context of the individual.
The present study was designed to track participants' eye movements during a dot-probe task in order to evaluate attentional biases to sensory pain-related words in individuals reporting chronic pain. On the basis of the literature reviewed above, five primary hypotheses were tested in the present study. In comparison to pain-free participants, individuals with chronic pain will: 1) exhibit a faster reaction time to sensory pain-related words in the dot-probe task; 2) attend to sensory pain-related words more frequently; 3) exhibit a different pattern of sustained attention to sensory pain-related and neutral words; 4) show an attentional bias toward sensory pain-related words at different stages of visual attentional processing; and 5) all participants will show significant, positive correlations between reaction time scores, visual attention measures, and self-reported pain-related psychological factors.

\section{Methods}

The study was approved by the York University Research Ethics Board (Human Participants Review Subcommittee). Written informed consent was obtained from participants before beginning the study.

\section{Recruitment}

Participants were recruited through York University's Undergraduate Research Participant Pool. Before participants enroll in studies, they complete a prescreening survey composed of questions submitted by faculty from various research laboratories. Responses to questions determine the studies that are visible to the student. For the present study, the question "do you experience physical pain on a regular basis?" was included to determine the approximate prevalence of chronic pain among undergraduate students completing the prescreen questionnaire; we defined chronic pain as the presence of ongoing pain that had persisted for 3 months or longer. ${ }^{28}$ Of the 3,163 students who responded, 655 (20.71\%) positively endorsed the statement, 2,508 (79.29\%) did not, and 26 declined to respond. Two study postings were created to recruit participants: one was visible to students who positively endorsed the prescreen question related to pain, and the other was visible only to students who did not. Participants received one course credit for completing the study. With this method, a total of 113 participants were recruited to participate and included in the final analysis.

\section{Materials}

\section{Hardware}

Eye movements were tracked using a Tobii T60 XL eye tracker with a 24-inch widescreen monitor and a $60 \mathrm{~Hz}$ data 
rate (Tobii Technology, Falls Church, VA, USA). The eye tracker is integrated into the monitor, allowing participants to move their heads freely throughout the study. The Tobii T60 $\mathrm{XL}$ has an accuracy rate of $0.5^{\circ}$ and $<0.03^{\circ} \mathrm{drift}$, reducing differences between what the participant is looking at and what the equipment is recording. Two Dell Precision T3400 Intel ${ }^{\circledR}$ Core $^{\mathrm{TM}} 2$ Quad CPUs with 4 GB of random-access memory (Dell Inc., Round Rock, TX, USA) were interfaced to facilitate data collection from two software programs, E-Prime 2.0 Professional (Psychology Software Tools, Inc., Sharpsburg, PA, USA) and Tobii Studio Professional 2.0 (Tobii Technology). The display resolution for the dot-probe protocol was $800 \times 600$ pixels to optimize video capture and data transfer. The study took place in a windowless room in order to standardize the lighting for all participants.

\section{Software}

E-Prime was used to design, display, collect, and prepare data for the dot-probe task. ${ }^{29}$ E-Prime provides millisecond precision in the capture of reaction times. The protocol was designed in E-Studio, data was combined with E-Merge, and data was prepared for export with E-DataAid. Tobii Studio Professional 2.0 captured participant eye movements with programmed time-sensitive markers. E-Prime and Tobii Studio software programs were interfaced with E-Prime 2.0 extensions for Tobii (Psychology Software Tools). Data were analyzed with SPSS version 20.0 (IBM Corporation, Armonk, NY, USA).

\section{Verbal stimuli}

Words were selected from previous research using the dotprobe task to test attentional biases in chronic pain populations. ${ }^{30-34}$ Words related to the sensory experience of pain were selected over words related to other aspects of the pain experience (eg, affective experience of pain, antecedents of pain, or consequences of pain), as sensory pain words were related to larger effects in previous research. ${ }^{8}$ Sensory pain words were originally drawn from the McGill Pain Questionnaire, a detailed questionnaire designed to describe diverse aspects of pain. ${ }^{35}$ Sensory pain and neutral words were matched for word length and frequency. ${ }^{36}$ See Table 1 for a complete list of word pairs.

\section{Behavioral measures}

During the dot-probe task, various behavioral measures were assessed. Reaction time (in milliseconds) was recorded to capture the speed of participant responses to the location of the dot probe. In addition, measures of visual attention were
Table I Word pairs

\begin{tabular}{ll}
\hline Sensory pain-neutral & Neutral-neutral \\
\hline Throbbing-blender & Bath-soap \\
Pounding-television & Brushing-decorate \\
Sharp-telephone & Clean-chair \\
Aching-door & Mugs-lamp \\
Burning-radio & Cook-dust \\
Dull-window & Decorated-household \\
Tender-stove & Dusted-plants \\
Sore-fur & Floor-steps \\
Gnawing-computer & Furniture-magazines \\
Hurting-pillow & Doorknob-bathroom \\
Shooting-table & Bedroom-surface \\
Stabbing-chair & Stair-table \\
Cramping-barrel & Water-house \\
Heavy-patio & Vase-tidy \\
Splitting-washer & Towels-bedspread \\
\hline
\end{tabular}

Note: Adapted from Asmundson G], Wright KD, Hadjistavropoulos HD. Hypervigilance and attentional fixedness in chronic musculoskeletal pain: consistency of findings across modified stroop and dot-probe tasks. J Pain. 2005;6(8):497-506, Copyright $\odot$ 2005, with permission from Elsevier. ${ }^{30}$

collected to determine the duration, frequency, and patterns of the participants' gaze.

\section{Reaction time measures}

For each participant, a congruency index, incongruency index, and neutral comparison index was computed. These indexes are based on calculations by Koster et a ${ }^{15}$ and Roelofs et al. ${ }^{37}$ A trial is considered to be congruent when the dot probe appears in the location on the screen where the target word (ie, the sensory pain word) was presented, and incongruent when the dot probe appears in the location of the neutral word (Figure 1). Each condition considers the location of the word and of the dot probe to balance individual differences in preference to look at one side of the computer screen more than the other, regardless of the location or type of word. The indexes are based on four mean reaction time (RT) scores, $\overline{R T}_{t d l r}, \overline{R T}_{t r d r}$, $\overline{R T}_{\text {trdl }}$, and $\overline{R T}_{t l d l}$, which are computed by taking the mean of the 15 trials in each condition. In each formula, " $t$ " is the target (sensory pain word), "d" is the dot probe, "l" is the left position on the screen, and " $\mathrm{r}$ " is the right position on the screen.

The congruency index is calculated by taking the grand mean of the mean RTs of congruent trials that are presented on the right and left sides of the computer screen: $\overline{R T}_{\text {congruent }}=\overline{R T}_{\text {tldl }}+\overline{R T}_{\text {trdr }} / 2$. Similarly, the incongruent index is calculated by taking the grand mean of the mean RTs of the responses of incongruent trials that are presented on the right and left sides of the computer screen: $\overline{R T}_{\text {incongruent }}=\overline{R T}_{\text {trdl }}+\overline{R T}_{\text {tldr }} / 2$. The neutral comparison index is calculated from the neutral-neutral 


\section{Congruency}

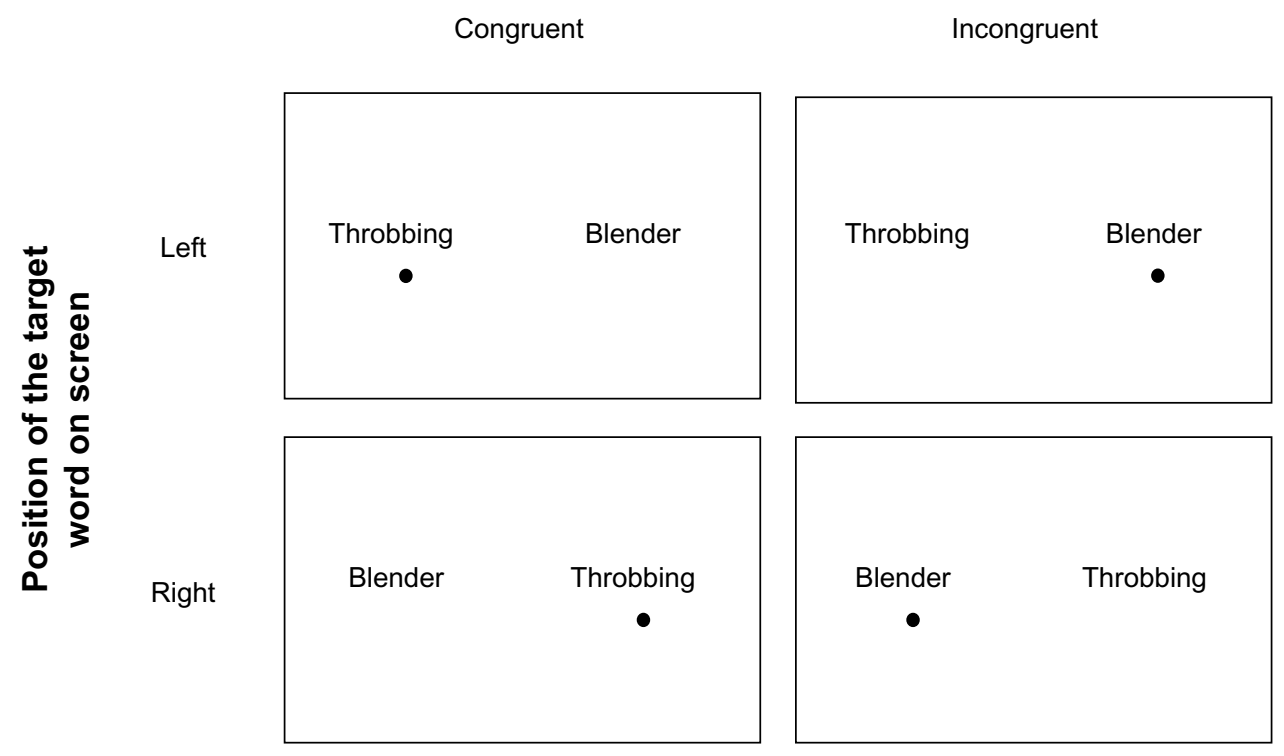

Figure I Visual depiction of counter-balanced trials of words presented during the dot-probe task.

Notes: Sensory pain word (target, t) on left (I) side of screen followed by the dot (d) on the left side of the screen (left, congruent or "tldl"), sensory pain word on left side of screen followed by the dot on the right ( $r$ ) side of the screen (left, incongruent or "tldr"), sensory pain word on right side of screen followed by the dot on the right side of the screen (right, congruent or "trdr"), and sensory pain word on right side of screen followed by the dot on the left side of the screen (right, incongruent or "trdl").

pairs and to permit comparison with the congruent and incongruent indexes. It is calculated by taking the grand mean of the mean reaction times for the neutral word pairs: $\overline{R T}_{\text {neutral }}=\overline{R T}_{\text {tldr }}+\overline{R T}_{\text {trdr }}+\overline{R T}_{\text {trdl }}+\overline{R T}_{\text {tldl }} / 4$.

\section{Visual attention measures}

Tobii Velocity-Threshold Identification (I-VT) fixation filter settings were used to classify fixations, as they provide validated and robust function parameter values that are easily replicated for future research. ${ }^{38}$ As such, maximum gap interpolation (ie, merging) of fixations was set to $75 \mathrm{~ms}$, the maximum angle between fixations was $0.5^{\circ}$, and fixations had to be greater than $60 \mathrm{~ms} .{ }^{39}$ These settings provide accurate fixation classifications for most research. ${ }^{39}$ Additionally, data was screened for validity and only used if the data collected was associated with the correct eye.

Gaze patterns were recorded for the duration of the dot-probe task. Currently, there are no accepted standard dependent variables associated with eye-tracking within a dot-probe task due to the novelty of its use. Therefore, we based our dependent variables in part on a recent study ${ }^{21}$ and in part on theoretical predictions. The following dependent variables were based on eye movements recorded within a region on the left and right sides of the screen defined by the experimenter as an "area of interest" (AOI). An AOI, 250 pixels in length by 107 pixels in height, was demarcated at mirror image regions on the (center and midline of the) left and right sides of the screen within which word stimuli were presented.

Two variables were calculated based on visual fixations for each word type (sensory pain and neutral): "number of fixations" was the number of times the participant's eyes focused on a sensory pain or neutral word within its respective AOI, and "average fixation duration" was the mean time associated with all fixations.

A "visit" was defined by one or more contiguous eyemovements within an AOI (ie, a visit ended when the eyes moved outside the AOI). Two variables were calculated on the basis of visits to the sensory pain word AOI or neutral word AOI. The "number of visits" was the total number of visits to the sensory pain word AOI or neutral word AOI, and "average visit duration" was the mean time spent looking during all visits to the sensory pain word AOI or neutral word AOI.

To investigate whether gaze patterns varied during different stages of visual processing/attention, we calculated the total fixation duration for three different periods of stimulus presentation: "early-phase total fixation duration" (0-500 ms); "middle-phase total fixation duration" (500-1,000 ms); and "late-phase total fixation duration" (1,000-2,000 ms). Each index was calculated by summing the total duration of each trial for each period. 


\section{Self-report measures}

Demographics and pain-history questionnaire

Participants were asked basic demographic questions and questions about their pain history and current pain complaints, including the duration, location, frequency, and cause of any pain. They were also asked to describe the pain intensity associated with several common types of pain (tooth ache, paper cut, stubbed toe, biting your tongue, sunburn, and ear ache) on a scale from $0-10$, where $0=$ no pain and $10=$ the highest pain intensity.

\section{Pain disability}

The Pain Disability Index (PDI) ${ }^{40}$ is a seven-item scale that assesses the degree of daily disability due to pain. Items are scored on an eleven-point Likert scale with a total scale score range of $0-70$, where higher scores reflect a greater degree of pain disability. The PDI has good internal consistency (Cronbach alpha $=0.86)$, good test-retest reliability $(r=0.44$ over a 2-month period), and good concurrent validity with measures of psychological distress, pain severity, and other items measuring pain-related disability. ${ }^{41}$ The internal consistency of the PDI in the present study was good (Cronbach alpha $=0.89$ ).

\section{Pain catastrophizing}

The Pain Catastrophizing Scale (PCS) $)^{42}$ is a 13 -item scale that measures the degree to which individuals catastrophize, ie, ruminate/worry about, magnify, and feel helpless in the face of painful experiences. Items are scored on a five-point Likert scale, and the total score range is $0-52$. Higher scores reflect more pain catastrophizing. Internal consistency is high (Cronbach alpha $=0.87$ ) and test-retest reliability is good $\left(r=0.75\right.$ over a 6 -week period). ${ }^{42}$ The internal consistency of the PCS in the present study was excellent (Cronbach alpha $=0.94)$.

\section{Pain vigilance}

The Pain Vigilance and Awareness Questionnaire (PVAQ) ${ }^{43}$ is a 16-item scale that assesses an individual's daily preoccupation with pain. Each item is scored on a five-point Likert scale, and the total score range is $0-64$. Higher scores indicate greater awareness, consciousness, vigilance, and observation of pain. The PVAQ has good internal consistency (Cronbach alpha $=0.86)$, good test-retest reliability $(r=0.80$ when retaken an average of 4 days later), and good convergent validity with private body consciousness $(r=0.58)$ and a negative correlation to the ignoring pain subscale of the Coping Strategies Questionnaire $(r=-0.24) .{ }^{43}$ The internal consistency of the PVAQ in the present study was good $($ Cronbach alpha $=0.87)$.

\section{Pain anxiety}

The Pain Anxiety Symptoms Scale - Short Form (PASS-SF) ${ }^{23}$ is a 20 -item scale that assesses anxiety regarding pain and pain sensations. Each item is scored on a five-point Likert scale, and the total score range is $0-100$. Higher scores reflect a tendency to be anxious about the experience of pain. The short form correlates highly with the original 40 -item scale $(r=0.97)$. The PASS-SF has excellent internal consistency (Cronbach alpha $=0.91)$, reliability, and validity (Cronbach alpha $=0.81) .{ }^{23}$ The internal consistency of the PASS-SF in the present study was excellent (Cronbach alpha $=0.93$ ).

\section{Chronic pain acceptance}

The Chronic Pain Acceptance Questionnaire (CPAQ) $)^{44}$ is a 20 -item scale that measures the tendency to accept the experience of pain and to engage in daily activities despite feeling pain. All items are scored on a seven-point Likert scale, and the total score range is $0-120$. Greater acceptance of chronic pain is reflected in higher scores. It has good internal consistency $($ Cronbach alpha $=0.85$ ), adequate reliability, and good convergent validity with related psychosocial distress and physical functioning questionnaires ${ }^{45}$ The internal consistency of the CPAQ in the present study was acceptable (Cronbach alpha $=0.74)$.

\section{State anxiety}

The state version of the State-Trait Anxiety Inventory $(\mathrm{STAI}-\mathrm{S})^{46}$ is a 20 -item scale that assesses current feelings of anxiety. Each item is rated on a four-point Likert scale, and the total score range is $20-80$. High scores are reflective of higher levels of state anxiety. Internal consistency is high (Cronbach alpha ranges from 0.86-0.95). Test-retest reliability is fairly low ( $r$ ranges from $0.16-0.62$ ) because state levels of anxiety are expected to change with time ${ }^{47}$ The STAI-S has well-established construct and concurrent validity. ${ }^{48}$ The internal consistency of the STAI-S in the present study was excellent (Cronbach alpha $=0.94)$.

\section{Anxiety sensitivity}

The Anxiety Sensitivity Index-3 (ASI-3) ${ }^{49}$ is an 18 -item questionnaire designed to assess beliefs and fear associated with anxiety-related sensations (specifically, that the symptoms of anxiety have harmful consequences). Items are scored on a five-point Likert scale, and the total score range is $0-72$. Higher scores reflect a greater tendency to be 
fearful of the experience of anxiety symptoms. The internal consistency for the subscales ranges from good to excellent (Cronbach alpha ranges from 0.80-0.90). The ASI-3 also has good reliability (Cronbach alpha $=0.93) .{ }^{50}$ The internal consistency of the ASI-3 in the present study was good (Cronbach alpha $=0.89)$.

\section{Illness sensitivity}

The Illness Sensitivity Index - Short Version (ISI-SF) ${ }^{51}$ is an eleven-item scale that assesses the fear of experiencing illness. Items are scored on a five-point Likert scale, and the total score range is $0-44$. Higher scores indicate a higher level of fear. The ISI has good internal consistency (Cronbach alpha $>0.80)^{51}$ and total scale reliability $(r=0.89)$. The internal consistency of the ISI-SF in the present study was excellent (Cronbach alpha $=0.91)$.

\section{Sensitivity to pain traumatization}

The Sensitivity to Pain Traumatization Scale (SPTS) ${ }^{25}$ is a 20-item measure of anxiety-related cognitive, emotional, and behavioral reactions to pain that resemble symptoms of posttraumatic stress disorder. Items are scored on a fivepoint Likert scale, and the total score range is $0-80$. The scale was developed with an exploratory factor analysis on the items of the PASS-SF, PCS, and ASI-3. The SPTS has good psychometric properties: the concurrent validity was established by comparing people with chronic pain and without chronic pain and it shows good convergent validity with related measures. ${ }^{25}$ The internal consistency of the SPTS in the present study was excellent (Cronbach alpha $=0.91$ ).

\section{Procedure}

After arriving at the lab and providing informed consent, participants completed a computer-administered survey consisting of the ten questionnaires described above. The demographic and pain history questions appeared at the beginning of the survey for all participants. The order of the remaining questionnaires was randomized for each participant using an online survey manager (Sona Systems, Bethesda, MD, USA). Participants were led to a windowless room to complete the dot-probe task. They were positioned approximately $60 \mathrm{~cm}$ in front of the screen with eyes level with the center of the screen. Five-point eye calibration was performed with Tobii Studio software to customize the eye-tracking specifications to each participant. The computer input was adjusted to run E-Prime from a second computer, whereupon a second eye calibration was performed. Next, the dot-probe task was introduced and described. Participants were told that they were to fix their gaze at the central fixation cross, that a pair of words would appear briefly on the screen and that a dot would be presented in the location of one of the words. Their task was to identify the location of the dot as quickly and accurately as possible. Participants then completed 12 practice trials to familiarize themselves with the task.

The protocol for each trial consisted of three parts: 1) a fixation cross ("+") appeared at the center of the screen for $500 \mathrm{~ms} ; 2$ ) two words in bold, Arial font in size 24 (sensory pain-neutral or neutral-neutral) were presented simultaneously, one on the left side and the other on the right side of the screen for 2,000 ms; and 3) immediately after the offset of the words, a dot probe (“•”) appeared on the left or right side of the screen (in the prior location of the word) for 2,000 ms or until the participant responded by pressing one of two keyboard keys. Participants were told to press the "P" with their right index finger if the dot probe was on the right side of the screen and the "Q" with their left index finger if the dot probe was on the left. All text and symbols appeared in black on a white background. Word pairs were counter-balanced, controlling for word type location and dot probe location, so that each word pair was presented four times (Figure 1). In total, participants completed 120 trials (four trials each of the 15 sensory pain-neutral word pairs and four trials each of the 15 neutral-neutral word pairs). The presentation order of trials was randomly generated by E-Prime (ie, the order selection was set to "random") for each participant.

\section{Statistical analysis}

Comparisons of nominal data categories, such as participants in the chronic pain group in comparison to pain-free participants, were made with chi-square tests of independence. The reaction time indexes were analyzed using a mixed design $2 \times 3$ analysis of variance (ANOVA) using group (chronic pain, pain-free) as the independent-samples factor and index type (congruent, incongruent, and neutral comparison indexes) as the within-subjects factor. Number of fixations, average fixation duration, number of visits, average visit duration, early-phase total fixation duration, middle-phase total fixation duration, and late-phase total fixation duration were analyzed by a series of $2 \times 2$ mixed-design ANOVAs using group (chronic pain, pain-free) as the between-subjects factor and word type (sensory pain, neutral) as the within-subjects factor. Significant interactions were proceeded with simple main effects analyses with the alpha criterion adjusted to $P=0.01$ to account for the number of comparisons. ${ }^{52}$ Correlation analyses were used to examine the strength of the linear 
relationships between total questionnaire scores and group, reaction time, and visual attention.

\section{Results}

\section{Demographic information}

As noted in the Recruitment section, the study was advertised to the 655 students who endorsed the prescreen statement "Do you experience pain on a regular basis?". Of these, 113 signed up and participated. We do not have data on why the remaining eligible participants did not sign up for the study. The age range of the sample of 113 participants was 18-44 years, mean $(\mathrm{M})_{\text {age }}=$ 21.32, standard deviation $(\mathrm{SD})_{\text {age }}=4.35$. Eighty-four participants were female $(74.30 \%)$ and 29 were male $(25.70 \%)$. The sample was ethnically diverse, with participants selfidentifying as Caucasian (32.54\%), South Asian (28.57\%), African (10.32\%), East Asian (9.53\%), Middle Eastern (8.73\%), Hispanic/Latino (4.76\%), Caribbean (3.97\%), Aboriginal $(0.79 \%)$, or undisclosed $(0.79 \%)$. Forty-nine participants wore vision-correction aids during testing, with 16 (14.2\%) using contact lenses and 33 (29.2\%) using glasses.

For the purpose of the present study, chronic pain was defined as the presence of ongoing pain that had persisted for 3 months or longer. ${ }^{28}$ According to this criterion, 51 participants $(45.13 \%)$ reported experiencing chronic pain. Of these, seven $(13.70 \%)$ reported experiencing pain for 3-6 months, five (9.80\%) for 6-12 months, and 39 (76.50\%) for 12 months or longer. Participants reported pain in one to five body locations $\left(\mathrm{M}_{\text {location }}=2.16, \mathrm{SD}_{\text {location }}=1.08\right): 30$ reported neck and/or back pain, 22 reported headache/migraine pain, 21 reported ankle and/or knee pain, 15 reported shoulder pain, 12 reported stomach pain, five reported hip(s) pain, two reported arm pain, one reported eye pain, one reported jaw pain, and one declined to respond to this question. Most participants reported that the pain commenced after an injury $(47.1 \%)$ or that they were unsure how the pain started (35.3\%), while $7.8 \%$ of participants stated that it started due to overuse and/or stress, $5.9 \%$ due to an illness, and one $(0.79 \%)$ due to a hereditary predisposition. Frequency of pain was reported as daily $(64.7 \%)$, weekly $(33.3 \%)$, or other $(2.0 \%)$ with the average intensity as mild (15.7\%), moderate $(74.5 \%)$, or severe $(9.8 \%)$. When completing the questionnaires, 30 participants reported their present pain intensity as mild $(73.3 \%)$ or moderate $(26.6 \%)$ pain. Approximately half $(52.9 \%)$ of chronic pain participants used painkillers on a regular basis. Of those participants, $63.0 \%$ used nonsteroidal anti-inflammatory drugs, $59.3 \%$ used acetaminophen, $25.9 \%$ used aspirin, 7.4\% used opioids, and 29.6\% reported using other types of painkiller.

Participants reporting pain $(n=51)$ did not differ significantly from those not reporting pain $(\mathrm{n}=62)$ on age, $t(111)=0.71, P=0.705$, sex, $\chi^{2}(2, \mathrm{n}=113)=0.001, P=0.969$, use of contact lenses, $\chi^{2}(1, \mathrm{n}=113)=0.014, P=0.905$, or glasses, $\chi^{2}(1, \mathrm{n}=113)=0.77, P=0.381$. Pearson chi-square $2 \times 4$ analysis using pain status (chronic pain, pain-free) and ethnicity (African descent, Asian descent, Caucasian, other) identified a significant difference according to ethnicity, $\chi^{2}(3, \mathrm{n}=113)=18.93, P<0.001$. Post-hoc comparisons showed a significantly greater proportion of participants self-identifying as Caucasian among those reporting pain.

\section{Data preparation}

\section{Reaction time measures}

On the dot-probe task, incorrect trials or trials that had reaction times $>2,000 \mathrm{~ms}$ were excluded from the calculation of the mean reaction time scores. ${ }^{15}$ Reaction times for these trials do not indicate a bias toward the measured variables and therefore may artificially skew the data. According to these criteria, $1.02 \%$ of trials were excluded from the calculation of the mean reaction time scores. Missing reaction time scores were prorated if $80 \%$ or more of trials were available. ${ }^{52,53}$ One participant with insufficient data was excluded from the analysis of the reaction time data.

\section{Visual attention measures}

Eye-tracking recordings were screened for the quality of visualgaze capture. Of the 135 participants recruited, only those whose gaze capture exceeded $75 \%$ were included, consistent with previous eye-tracking research. ${ }^{4}$ Lower gaze capture may be related to compromised attention, consistently lowered/closed eyelids, or reflections off of glasses that obscure the visual recording. ${ }^{55}$ This led to the exclusion of 20 participants with poor capture $\left(\mathrm{M}_{\text {capture }}=60.90 \%, \mathrm{SD}_{\text {capture }}=12.52 \%\right.$ ) and two participants who were unable to be calibrated. The mean eye capture for included participants $(n=113$ ) was $88.44 \%, \mathrm{SD}=5.34 \%$.

Participants included in the final analysis did not differ significantly from those with insufficient gaze data $(\mathrm{n}=22)$ on age, $t(133)=0.71, P=0.481$, use of contact lenses, $\chi^{2}(1, \mathrm{n}=135)=5.50, P=0.064$, glasses, $\chi^{2}(1, \mathrm{n}=135)=0.45$, $P=0.504$, or presence of chronic pain, $\chi^{2}(1, \mathrm{n}=135)=0.58$, $P=0.448$. There was a significantly greater proportion of males, $\chi^{2}(1, \mathrm{n}=135)=11.84, P=0.003$. Pearson chi-square $2 \times 4$ analysis using pain status (chronic pain, pain-free) and ethnicity (African descent, Asian descent, Caucasian, other) identified a significant difference according to ethnicity, 
$\chi^{2}(3, \mathrm{n}=135)=7.749, P=0.051$. Post-hoc comparisons showed a significantly greater proportion of participants self-identifying as Caucasian among those with sufficient gaze data.

\section{Questionnaires}

Missing questionnaire items were prorated if $80 \%$ or more of trials were available. ${ }^{53,54}$ One participant responded to fewer than $80 \%$ of the questions on the ASI-3, STAI-S, and SPTS and was consequently excluded from the analysis of these questionnaires.

\section{Normality}

There was no evidence that assumptions of normality were violated in the present data set: sphericity was not violated according to Box's Test of Equality of Covariance Matrices and Mauchly's Test of Sphericity. Levene's Test of Equality of Error Variances did not show evidence that homogeneity of variance was violated. Shapiro-Wilk Test of Normality and an examination of skewness and kurtosis according to a test statistic adapted from $\mathrm{Cramer}^{56}$ (sample skewness divided by the standard error of skewness), did not suggest violations in normality. Exceptions for any tests are discussed in conjunction with the specific analysis below.

\section{Hypothesis I: dot-probe task reaction time measures}

Table 2 shows the mean reaction times for the congruency, incongruency, and neutral indexes according to chronic pain and pain-free participants. A mixed-design ANOVA evaluated the relationship between group and index type (congruency, incongruency, and neutral comparison index). Mauchly's Test of Sphericity was significant, $\chi^{2}(2)=9.30, P=0.01$ which necessitated a Huynh-Feldt correction. ${ }^{57}$ Significant effects were not found for group, $F(1,110)=0.59, P=0.451$, $\eta_{\mathrm{p}}{ }^{2}=0.012$, index type, $F(1.90,208.57)=2.27, P=0.113$, $\eta_{\mathrm{p}}{ }^{2}=0.021$, or the group by index type interaction, $F(1.90$, 208.57) $=0.70, P=0.490, \eta_{\mathrm{p}}^{2}=0.006$.

Table 2 Hypothesis I: reaction time index scores (in milliseconds) for the chronic pain group $(n=5 \mathrm{I})$ and the pain-free group $(n=62)$

\begin{tabular}{lll}
\hline & $\begin{array}{l}\text { Chronic pain group, } \\
\text { mean (SD) }\end{array}$ & $\begin{array}{l}\text { Pain-free group, } \\
\text { mean (SD) }\end{array}$ \\
\hline Congruency index & $532.18(93.58)$ & $548.15(88.80)$ \\
Incongruency index & $526.76(84.96)$ & $539.28(79.12)$ \\
Neutral comparison index & $532.69(85.82)$ & $540.37(86.78)$ \\
\hline
\end{tabular}

Note: Significant effects were not found for group, index type, or group by index type interaction.

Abbreviation: SD, standard deviation.

\section{Hypothesis 2: frequency measures of visual attention using eye-tracking}

Table 3 shows the means and standard deviations for the visual attention measures by group. Mixed-design ANOVAs evaluated differences between group (chronic pain versus pain free) and word type (sensory pain versus neutral) for all visual attention measures. For number of fixations, the main effect of group, $F(1,111)=4.55, P=0.035, \eta_{\mathrm{p}}{ }^{2}=0.039$, word type, $F(1,111)=66.03, P<0.001, \eta_{\mathrm{p}}{ }^{2}=0.373$, and the group by word type interaction were significant, $F(1,111)=6.06, P=0.015$, $\eta_{\mathrm{p}}{ }^{2}=0.052$. Simple main effects showed that the number of fixations on threat words was greater for participants with chronic pain than for pain-free participants, $F(1,111)=6.46$, $P=0.012, \eta_{\mathrm{p}}{ }^{2}=0.055$, and the number of fixations on sensory pain words was significantly greater than that for neutral words among pain-free participants, $F(1,111)=17.77, P<0.001$, $\eta_{\mathrm{p}}{ }^{2}=0.138$, and for chronic pain participants, $F(1,111)=51.07$, $P<0.001, \eta_{\mathrm{p}}^{2}=0.315$ (Figure 2).

For number of visits, only the main effect of word type was significant, $F(1,111)=34.37, P<0.001, \eta_{\mathrm{p}}{ }^{2}=0.236$ [group: $F(1,111)=1.64, P=0.204, \eta_{\mathrm{p}}{ }^{2}=0.015$, group by word type interaction: $\left.F(1,111)=2.21, P=0.140, \eta_{\mathrm{p}}{ }^{2}=0.020\right]$.

\section{Hypothesis 3: measures of sustained visual attention using eye-tracking}

For average fixation duration, none of the effects was significant [group: $F(1,111)=2.93, P=0.090, \eta_{\mathrm{p}}{ }^{2}=0.026$, word type: $F(1,111)=0.41, P=0.523, \eta_{\mathrm{p}}{ }^{2}=0.004$, group by word type interaction: $\left.F(1,111)=1.16, P=0.283, \eta_{\mathrm{p}}{ }^{2}=0.010\right]$.

For average visit duration, the main effect of word type, $F(1,111)=22.34, P<0.001, \eta_{\mathrm{p}}{ }^{2}=0.168$, and the group by word type interaction were significant, $F(1,111)=4.15, P=0.044$, $\eta_{\mathrm{p}}{ }^{2}=0.036$ [group: $F(1,111)=0.03, P=0.876, \eta_{\mathrm{p}}{ }^{2}=0.000$ ] Simple main effects showed that the average visit duration for sensory pain words was significantly longer than that for neutral words among participants with chronic pain, $F(1,111)=20.839, P<0.001, \eta_{\mathrm{p}}{ }^{2}=0.158$ (Figure 3).

\section{Hypothesis 4: measures of visual attention according to presentation phase}

The pattern of results was the same for the early-phase and middle-phase total fixation duration. For early-phase total fixation duration, only the main effect of word type was significant, $F(1,111)=8.24, P=0.005, \eta_{\mathrm{p}}^{2}=0.069$ [group: $F(1,111)=3.42$, $P=0.067, \eta_{\mathrm{p}}{ }^{2}=0.030$, group by word type interaction: $\left.F(1,111)=0.28, P=0.599, \eta_{\mathrm{p}}{ }^{2}=0.002\right]$. Similarly, for middle- 
Table 3 Hypotheses 2-4: frequency and duration of eye-tracking dependent variables for the chronic pain group ( $\mathrm{n}=5 \mathrm{I}$ ) and the painfree group $(n=62)$

\begin{tabular}{|c|c|c|c|c|}
\hline & \multicolumn{2}{|l|}{ Chronic pain group } & \multicolumn{2}{|l|}{ Pain-free group } \\
\hline & $\begin{array}{l}\text { Sensory pain words, } \\
\text { mean (SD) }\end{array}$ & $\begin{array}{l}\text { Neutral words, } \\
\text { mean (SD) }\end{array}$ & $\begin{array}{l}\text { Sensory pain words, } \\
\text { mean (SD) }\end{array}$ & $\begin{array}{l}\text { Neutral words, } \\
\text { mean (SD) }\end{array}$ \\
\hline \multicolumn{5}{|l|}{ Hypothesis 2: frequency of visual attention } \\
\hline Number of fixations $s^{a-c}$ & |94.3| (40.37) & $172.10(34.75)$ & $|72.8|(48.06)$ & $160.92(42.27)$ \\
\hline Number of visits ${ }^{a}$ & $|23.4|(22.4 \mid)$ & II $5.39(21.05)$ & I I $5.84(22.58)$ & $111.06(26.62)$ \\
\hline \multicolumn{5}{|l|}{ Hypothesis 3: sustained visual attention } \\
\hline Average fixation duration (milliseconds) & $0.195(0.04)$ & $0.192(0.04)$ & $0.206(0.04)$ & $0.207(0.04)$ \\
\hline Average visit duration (milliseconds) $)^{\mathrm{a}, \mathrm{c}}$ & $0.334(0.06)$ & $0.310(0.06)$ & $0.329(0.06)$ & $0.319(0.06)$ \\
\hline \multicolumn{5}{|l|}{ Hypothesis 4: presentation phase } \\
\hline Early-phase total fixation duration $(0-500 \mathrm{~ms})^{\mathrm{a}}$ & $6.27(2.02)$ & $5.89(1.93)$ & $5.56(2.14)$ & $5.30(1.73)$ \\
\hline Middle-phase total fixation duration $(500-1,000 \mathrm{~ms})^{\mathrm{a}}$ & $10.31(2.67)$ & $8.78(2.40)$ & $9.90(2.82)$ & $8.86(2.5 \mathrm{I})$ \\
\hline Late-phase total fixation duration $(\mathrm{I}, 000-2,000 \mathrm{~ms})^{\mathrm{a}, \mathrm{c}}$ & $21.46(7.09)$ & I8.37 (5.37) & $19.53(6.06)$ & $18.9 \mid(6.34)$ \\
\hline
\end{tabular}

Notes: andicates a significant main effect of word type; bindicates a significant main effect of group; cindicates a significant group by word type interaction.

Abbreviation: SD, standard deviation.

phase total fixation duration only the main effect of word type was significant, $F(1,111)=44.10, P<0.001, \eta_{\mathrm{p}}^{2}=0.284$ [group: $F(1,111)=0.13, P=0.721, \eta_{\mathrm{p}}^{2}=0.001$, group by word type interaction: $\left.F(1,111)=1.58, P=0.211, \eta_{\mathrm{p}}^{2}=0.014\right]$.

In contrast, for late-phase total fixation duration, the main effect of word type, $F(1,111)=12.59, P=0.001$, $\eta_{\mathrm{p}}{ }^{2}=0.102$, and the group by word type interaction were significant, $F(1,111)=5.594, P=0.020, \eta_{\mathrm{p}}{ }^{2}=0.048$ [group: $\left.F(1,111)=0.436, P=0.510, \eta_{\mathrm{p}}^{2}=0.004\right]$. Simple main effects showed that the total late-phase fixation duration for sensory pain words was significantly longer than that for neutral

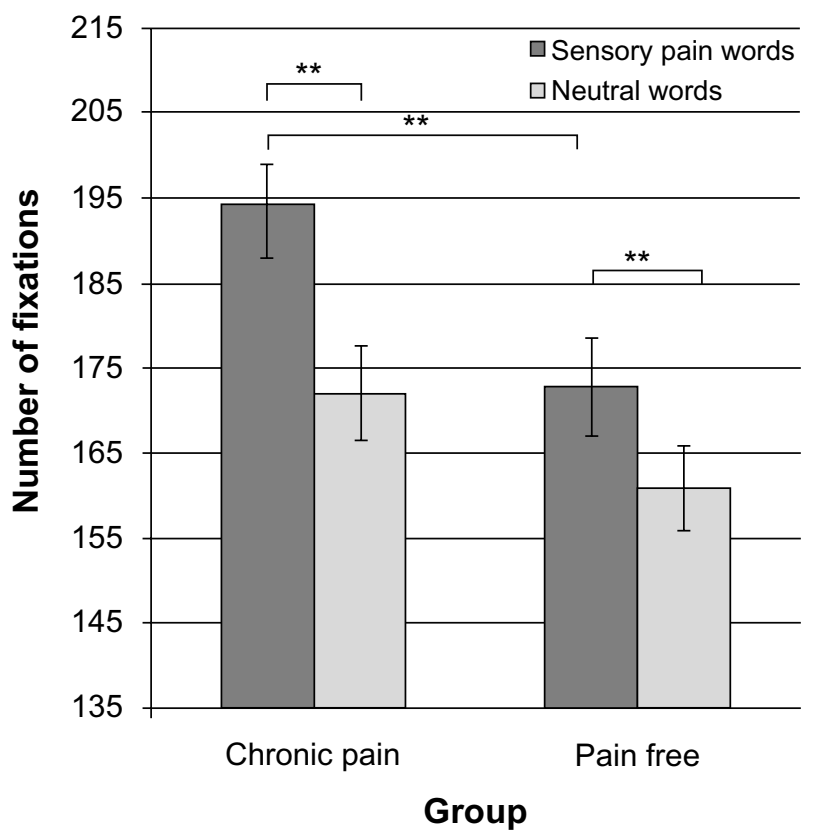

Figure 2 Frequency of fixations for each group. Notes: Error bars display the standard error. $* * p<0.01$. words among participants with chronic pain, $F(1,111)=15.94$, $P<0.001, \eta_{\mathrm{p}}{ }^{2}=0.126$ (Figure 4).

\section{Hypothesis 5: relationship between questionnaires and behavioral measures Group effects}

Table 4 shows the means and SDs for the questionnaire total scores for participants with and without chronic pain. Groups were compared with Student's $t$-tests, and the Type I error rate was adjusted to $P=0.006$ using a Bonferroni correction to adjust for alpha error rate inflation. ${ }^{52} \mathrm{Chronic}$ pain partici-

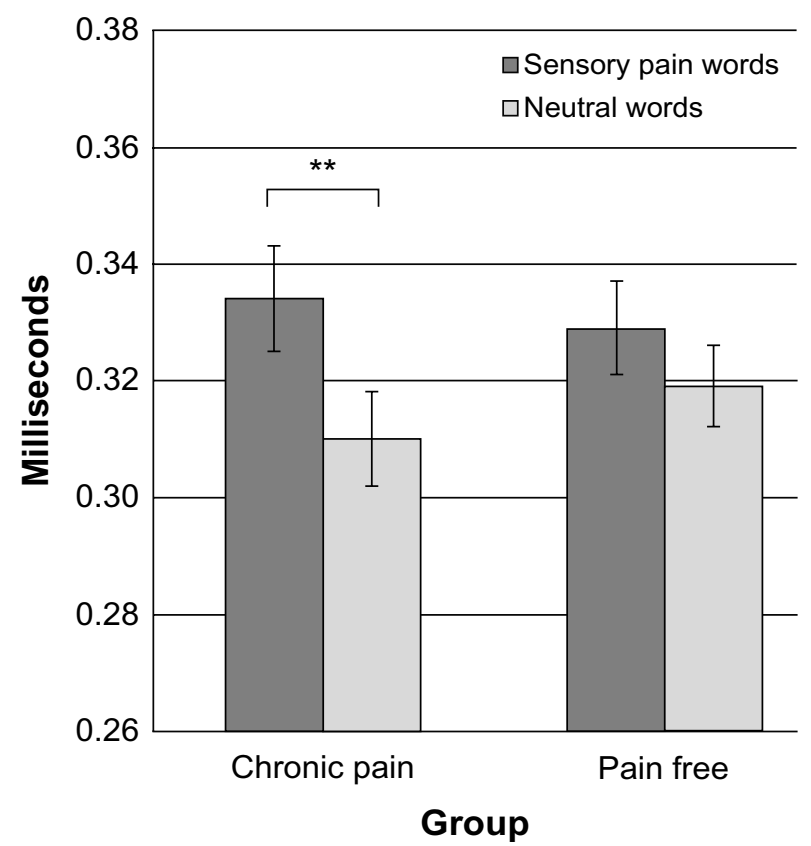

Figure 3 Average visit duration for each group. Notes: Error bars display the standard error. $* * p<0.01$. 


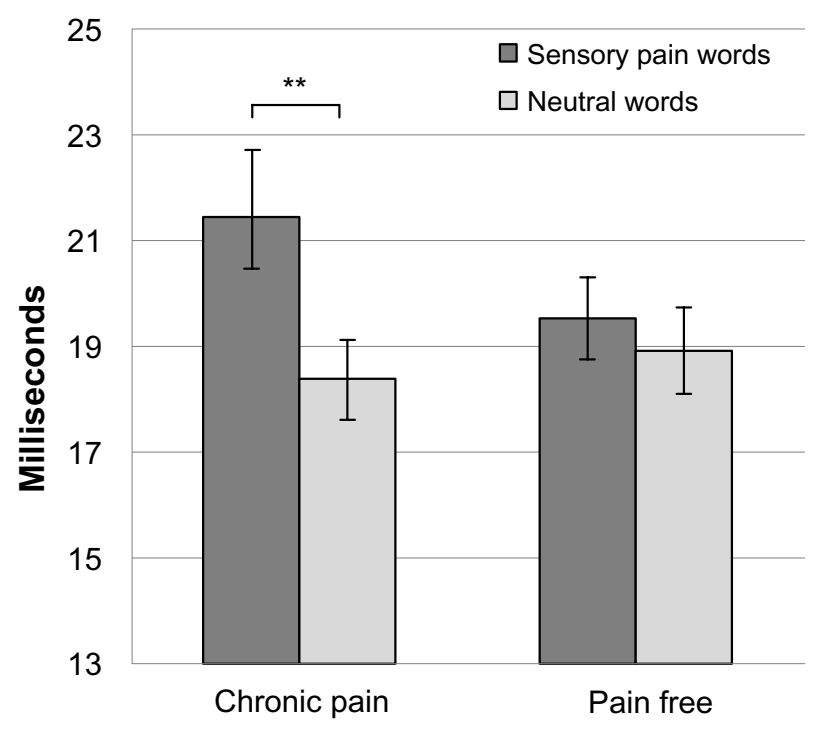

\section{Group}

Figure 4 Late-phase total fixation duration for each group. Notes: Error bars display the standard error. $* * p<0.01$.

pants reported significantly higher levels of pain disability and chronic pain acceptance.

\section{Correlations among reaction time and visual attention measures}

Pearson correlations evaluated the relationship between questionnaires and behavioral measures. Only correlations with sensory pain words were examined. The Type I error rate was adjusted to $P=0.003$ for reaction time measures and

Table 4 Hypothesis 5: comparisons between the chronic pain group $(n=5 I)$ and pain-free group $(n=62)$ according to questionnaire data

\begin{tabular}{|c|c|c|c|c|}
\hline Scale & $\begin{array}{l}\text { Chronic } \\
\text { pain group, } \\
\text { mean (SD) }\end{array}$ & $\begin{array}{l}\text { Pain-free } \\
\text { group, } \\
\text { mean (SD) }\end{array}$ & $t(d f)$ & $P$ \\
\hline PDI & $20.18(12.00)$ & $5.37(9.21)$ & $-7.42(92.4 I)^{\mathrm{a}}$ & $<0.001 *$ \\
\hline PCS & $19.22(11.69)$ & $16.14(12.00)$ & $-1.36(111)$ & 0.176 \\
\hline PVAQ & $46.18(10.69)$ & $41.23(9.64)$ & $-2.59(\mathrm{III})$ & 0.011 \\
\hline PASS-SF & $37.72(\mid 7.6 I)$ & $36.03(19.94)$ & $-0.474(\mathrm{III})$ & 0.637 \\
\hline CPAQ & 77.57 (I4.74) & $66.57(14.74)$ & $-4.15(111)$ & $<0.00 I^{*}$ \\
\hline STAI-S & 39.53 (I2.39) & 34.01 (11.14) & $-2.49(110)$ & 0.014 \\
\hline ASI-3 & $21.49(12.86)$ & $22.36(13.33)$ & $0.349(110)$ & 0.728 \\
\hline ISI-SF & I6.7I (8.64) & I6.23 (9.55)) & $-0.277(\mathrm{III})$ & 0.782 \\
\hline SPTS & 27.91 (13.48) & 26.93 (I5.39) & $-0.356(\mathrm{I} 10)$ & 0.722 \\
\hline
\end{tabular}

Notes: 'Adjusted Student's $t$-test score reported due to a violation in Levene's Test of Equality of Variance, $F(2,108)=5.75, P=0.018$; $*$ indicates a significant difference in scores at an adjusted $P=0.006$ to correct for multiple comparisons (Bonferroni).

Abbreviations: SD, standard deviation; df, degrees of freedom; PDI, Pain Disability Index; PCS, Pain Catastrophizing Scale; PVAQ, Pain Vigilance and Awareness Questionnaire; PASS-SF, Pain Anxiety Symptoms Scale - Short Form; CPAQ, Chronic Pain Acceptance Questionnaire; STAI-S, State-Trait Anxiety Inventory (state version); ASI-3, Anxiety Sensitivity Index-3; ISI-SF, Illness Sensitivity IndexShort Version; SPTS, Sensitivity to Pain Traumatization Scale. to $P=0.001$ for visual attention measures with a Bonferroni correction to control error associated with the large number of comparisons. ${ }^{52}$ One-tailed tests were used, consistent with predicted outcomes. Five correlations were significant for participants with chronic pain: PCS total score and incongruency index, $r(51)=0.38, P=0.003$, CPAQ total score and congruency index, $r(51)=-0.39, P=0.002$, CPAQ total score and incongruency index, $r(51)=-0.38, P=0.003$, and ISI-SF total score and late-phase total fixation duration, $r(51)=0.482$, $P<0.001$. Only the correlation the STAI-S total score and early-phase total fixation duration was significant for painfree participants, $r(61)=-0.38, P=0.001$.

\section{Discussion}

The present study used eye-tracking technology to compare attentional biases to threat versus neutral words in individuals with and without chronic pain using a dot-probe task. Significant differences were not found for reaction time. In contrast, significant group by word type interaction effects were found for several eye-tracking measures: number of fixations, average visit duration, and late-phase total fixation duration. These findings indicate that individuals with chronic pain, but not those who are pain-free, display a bias toward sensory pain words (Figures 3 and 4) and that individuals with chronic pain differ from those who are pain-free in terms of how frequently they attend to sensory pain words (Figure 2). Taken together, these findings suggest that eyetracking technology provides more sensitive measures of a bias than does the dot-probe task.

As noted above, dot-probe reaction times did not show evidence of an attentional bias, contrary to the expectations of our first hypothesis (Table 2). There are several possible explanations for the absence of an effect. The most likely one is the limitation associated with using reaction time as the sole measure of attention. ${ }^{12,58}$ Reaction time is an indirect indicator of visual attention, where faster congruent reaction times suggest that the participant's gaze is on the threat word before the dot appears on the screen, ${ }^{10}$ creating a substantial margin of error. Another possibility is that the exposure duration used in the present study was longer than that in other studies. ${ }^{2}$ To our knowledge, only one other study has used an exposure duration of 2,000 $\mathrm{ms}$ in a sample of people with pain, and the results also failed to show significant differences. ${ }^{11}$ However, this explanation does not appear to account for the lack of differences in reaction time, since a recent meta-analysis ${ }^{8}$ of dot-probe studies showed larger effect sizes associated with exposure durations longer than $1,000 \mathrm{~ms}$ in comparison to exposure durations between 
0-500 ms and 500-1,000 ms. Another possibility may be the nature of the sample used in the present study; namely, students who self-reported experiencing chronic pain. Attentional biases have been more consistently identified with reaction times using the dot-probe task in individuals recruited from clinical settings. ${ }^{37,59,60}$ Individuals seeking treatment for pain likely experience greater distress and preoccupation with painful sensations, leading to greater attentional biases that then become detectable with the dotprobe task. This suggests that only substantial attentional biases can be identified with reaction time as the outcome measure, whereas smaller biases can be identified with eyetracking, as in the present study.

In contrast to the lack of findings using reaction time as the bias measure, number of fixations, average visit duration, and late-phase total fixation duration showed evidence for a bias toward pain-related stimuli in individuals with chronic pain, supporting hypotheses 2-4. Of particular interest is the finding that a bias to sensory pain words, as measured by fixation duration, was only evident in the later stage of attentional processing and not the early or middle stages (Figure 4). Because later stages of attentional processing are associated with more top-down, conscious processes, such as avoidance and re-engagement, ${ }^{17,18}$ this supports the proposition that increased attention toward pain-related stimuli reflects more of an explicit, cognitive bias rather than an automatic, preattentive bias. ${ }^{2,8}$ That is, the bias becomes evident only once the threat has been identified and its meaning and relevance have become apparent. The late-phase findings are consistent with the work of Pincus and Morley ${ }^{6}$ who proposed the presence of an explicit cognitive-processing bias in chronic pain.

In contrast, number of fixations and average visit duration (Figures 2 and 3) were calculated across the course of each trial (ie, 0-2,000 ms) so that the significant interactions may reflect a bias in any one or more of the following phases of attention: initial orientation and engagement, maintenance, or re-engagement. ${ }^{2,13-16,18}$ Given the simplicity of the dotprobe task, it may be that the associated attentional processing required by participants was not sufficiently intentional and conscious to be considered controlled, but the present results do not permit a conclusion regarding the stage(s) of processing that underlies the present effects. Nevertheless, the results clearly show that participants with chronic pain fixated more frequently on pain words than did pain-free participants (Figure 2) and that the average visit duration for sensory pain words was significantly longer than that for neutral words among participants with chronic pain (Figure 3). Future research might consider a finer-grained analysis to determine the extent to which these significant effects are guided by early (eg, bottom-up, preattentive) or later (top-down, cognitive) processes.

Eye-tracking measures also detected significant word type and group by word type interaction effects (Table 3 ) independent of pain status. Word type effects showed that all participants looked more frequently at and for longer durations on sensory pain words than neutral words. This supports the idea that humans are evolutionarily predisposed to attend to threats in the environment: automatic processing provides information regarding the threat to prepare for a behavioral response, such as escape or confrontation. ${ }^{60-62}$ Because sensory pain words have a higher threat value than neutral words, this may have led to increased attention by all participants.

We expected to find significant correlations among reaction time scores, visual attention measures, and self-reported pain-related psychological factors. For participants with chronic pain, only five correlations were significant, providing provisional support for hypothesis 5 . We found that higher pain catastrophizing scores were related to higher incongruency scores. As high incongruency scores reflect longer response times to trials in which the dot appears behind the neutral words, this suggests that participants high in catastrophizing have a bias away from neutral words. This is consistent with the increased level of vigilance proposed in individuals that catastrophize. ${ }^{42,64}$ Both the congruency and incongruency indexes were negatively related to chronic pain acceptance scores, suggesting that participants higher in chronic pain acceptance respond faster on all trials, regardless of the location of the dot. Since pain has been linked to diminished performance on cognitive tasks ${ }^{65,66}$ as a form of interruption, ${ }^{1}$ it is possible that greater levels of acceptance of the pain experience increased the level of performance in the dot-probe task. The finding that participants with higher illness sensitivity scores spent more time looking at threat words during the late-phase for total fixation duration shows that the more fearful a participant is of experiencing illness, the more likely they are to attend to pain-related stimuli. Overall, there is some evidence to support the hypothesis of a relationship between pain-related psychological factors and attentional patterns among participants with chronic pain.

The present study has important clinical implications regarding the development and course of chronic pain. According to the Fear-Avoidance Model, increased attention toward pain sensations can exacerbate symptoms, leading to avoidance of activities that produce pain, physical deconditioning, and consequently more pain. ${ }^{67}$ In the Misdirected 
Problem-Solving Model, increasing attention to pain-related stimuli disrupts routine cognitive functioning and promotes worry, motivating the individual with pain to engage in problem-solving behavior (eg, taking medication, seeing the doctor) ${ }^{68}$ However, if the pain persists after problem-solving behavior, the attentional activation continues to contribute to the cycle of worry, hypervigilance, and pain interruption, thereby worsening outcomes. This model suggests that acceptance of chronic pain, therefore, can reduce psychological symptoms such as distress, anxiety, and depression, as well as physical symptoms such as pain and disability. ${ }^{69}$ This is supported by the finding that increased levels of chronic pain acceptance are associated with a reduced attentional bias toward pain-related information. ${ }^{70}$ Therefore, if attentional biases causally contribute to the development and maintenance of chronic pain as has been proposed, then eye-tracking technology can be used to monitor progress and efficacy of pain-management interventions.

The present study had several limitations. First, due to programming limitations, we were unable to collect data related to the direction of first fixation and first fixation duration for each trial, an index used in previous research. ${ }^{11,21}$ This would have captured early attention more effectively. ${ }^{4}$ Second, the present study used words related to the sensory aspects of pain to detect attentional biases. Words reflect a semantic understanding of threat and may not adequately capture automatic processing responses that people with chronic pain experience. ${ }^{1,7}$ Future studies should consider using pictures related to pain (eg, images of others experiencing pain, injuries, or objects that may cause pain) to increase the ecological validity of the visual stimuli. Third, the chronic pain group was heterogeneous: pain was experienced in a wide variety of body locations (most notably the neck/back and headache/migraine pain) and the frequency and severity of pain was varied. It is possible that the presence and direction of attentional biases may differ according to these qualities. This is consistent with the finding that attentional biases, at least as measured by the traditional dot-probe task, are not found in populations that experience procedural pain, acute pain, and experimental pain. ${ }^{8}$ Fourth, this study did not include a comprehensive assessment of the participants' medical treatment or psychiatric history. Since attentional biases have been more consistently identified in clinical-pain populations ${ }^{37,59,60}$ and in individuals diagnosed with anxiety disorders, ${ }^{71}$ these variables may have contributed to the present findings. Relatedly, pain and psychopharmacological medication may also contribute to reaction time and gaze-duration variables: if the experience of pain was less salient during study testing due to medication, this may have led to smaller attentional biases. Future studies should perform a comprehensive assessment of the medical and psychiatric history of participants and control for medication use.

A final relevant issue is related to the processing of threatrelated material. Most studies using the dot-probe investigate attentional biases toward pain-related information. However, it is possible that some people display a bias away from painrelated stimuli, as predicted by the fear-avoidance model of chronic pain. ${ }^{72}$ If this were the case, it is possible that the absence of an overall effect of an attentional bias reported in many studies actually reflects a bias toward pain-related stimuli in some individuals and a bias away from pain-related stimuli in others. This pattern of results would mask the effects of the two biases that operate in opposite directions and would produce an overall nonsignificant finding. Future studies should evaluate whether, in whom, and under what circumstances biases are evident toward and away from pain-related stimuli.

In summary, the present study used eye-tracking methodology to investigate attentional biases toward sensory pain words in individuals with and without chronic pain. The results showed that all participants attended to stimuli related to pain more than to neutral stimuli and that this effect was more pronounced among participants with chronic pain as measured by number of fixations, average visit duration, and late-phase total fixation duration. As well, a larger effect was present in later stages of attentional processing, suggesting the presence of a top-down cognitive bias toward sensory pain words. In contrast, the traditional dot-probe reaction time measures did not show evidence for an attentional bias. Taken together, these findings suggest that eye-tracking technology provides a more accurate and sensitive measure of selective visual attentional biases than reaction time.

\section{Acknowledgments}

Samantha Fashler is supported by an Ontario Graduate Scholarship and a Canadian Institutes of Health Research (CIHR) Frederick Banting and Charles Best CGS Master's Award. Joel Katz is supported by a CIHR Canada Research Chair (CRC) in Health Psychology. Funds to support the project were obtained from Dr Katz's CRC. We are grateful to Dr. Paul Ritvo for providing partial funding for the purchase of the Tobii eye-tracker through a Federal Development Grant. This article was derived, in part, from Samantha Fashler's master's thesis. 


\section{Disclosure}

The authors report no conflicts of interest in this work.

\section{References}

1. Eccleston C, Crombez G. Pain demands attention: a cognitiveaffective model of the interruptive function of pain. Psychol Bull. 1999;125(3):356-366.

2. Schoth DE, Nunes VD, Liossi C. Attentional bias towards painrelated information in chronic pain; a meta-analysis of visual-probe investigations. Clin Psychol Rev. 2012;32(1):13-25.

3. Liossi C, Schoth DE, Godwin HJ, Liversedge SP. Using eye movements to investigate selective attention in chronic daily headache. Pain. 2014;155(3):503-510.

4. Vervoort T, Trost Z, Prkachin KM, Mueller SC. Attentional processing of other's facial display of pain: an eye tracking study. Pain. 2013;154(6):836-844.

5. Van Damme S, Crombez G, Eccleston C. Disengagement from pain: the role of catastrophic thinking about pain. Pain. 2004;107(1-2): 70-76.

6. Pincus T, Morley S. Cognitive-processing bias in chronic pain: a review and integration. Psychol Bull. 2001;127(5):599-617.

7. Asmundson GJ, Carleton RN, Ekong J. Dot-probe evaluation of selective attentional processing of pain cues in patients with chronic headaches. Pain. 2005;114(1-2):250-256.

8. Crombez G, Van Ryckeghem DM, Eccleston C, Van Damme S. Attentional bias to pain-related information: a meta-analysis. Pain. 2013;154(4):497-510.

9. Dear BF, Sharpe L, Nicholas MK, Refshauge K. The psychometric properties of the dot-probe paradigm when used in pain-related attentional bias research. J Pain. 2011;12(12):1247-1254.

10. MacLeod C, Mathews A, Tata P. Attentional bias in emotional disorders. J Abnorm Psychol. 1986;95(1):15-20.

11. Yang Z, Jackson T, Chen H. Effects of chronic pain and pain-related fear on orienting and maintenance of attention: an eye movement study. J Pain. 2013;14(10):1148-1157.

12. Sharpe L. Attentional biases in pain: more complex than originally thought? Pain. 2014;155(3):439-440.

13. Bradley BP, Mogg K, Millar NH. Covert and overt orienting of attention to emotional faces in anxiety. Cogn Emot. 2000;14(6):789-808.

14. Gamble AL, Rapee RM. The time-course of attentional bias in anxious children and adolescents. J Anxiety Disord. 2009;23(7):841-847.

15. Koster EH, Crombez G, Verschuere B, De Houwer J. Selective attention to threat in the dot probe paradigm: differentiating vigilance and difficulty to disengage. Behav Res Ther. 2004;42(10):1183-1192.

16. Koster EH, Verschuere B, Crombez G, Van Damme S. Time-course of attention for threatening pictures in high and low trait anxiety. Behav Res Ther. 2005;43(8):1087-1098.

17. Hermans D, Vansteenwegen D, Eelen P. Eye movement registration as a continuous index of attention deployment: data from a group of spider anxious students. Cogn Emot. 1999;13(4):419-434.

18. Heeren A, Lievens L, Philippot P. How does attention training work in social phobia: disengagement from threat or re-engagement to nonthreat? J Anxiety Disord. 2011;25(8):1108-1115.

19. Gatchel RJ, Peng YB, Peters ML, Fuchs PN, Turk DC. The biopsychosocial approach to chronic pain: scientific advances and future directions. Psychol Bull. 2007;133(4):581-624.

20. Picavet HS, Vlaeyen JW, Schouten JS. Pain catastrophizing and kinesiophobia: predictors of chronic low back pain. Am J Epidemiol. 2002;156(11):1028-1034.

21. Yang Z, Jackson T, Gao X, Chen H. Identifying selective visual attention biases related to fear of pain by tracking eye movements within a dot-probe paradigm. Pain. 2012;153(8):1742-1748.

22. Zvolensky MJ, Goodie JL, McNeil DW, Sperry JA, Sorrell JT. Anxiety sensitivity in the prediction of pain-related fear and anxiety in a heterogeneous chronic pain population. Behav Res Ther. 2001;39(6):683-696.
23. McCracken LM, Dhingra L. A short version of the Pain Anxiety Symptoms Scale (PASS-20): preliminary development and validity. Pain Res Manag. 2002;7(1):45-50.

24. Asmundson GJ, Katz J. Understanding the co-occurrence of anxiety disorders and chronic pain: state-of-the-art. Depress Anxiety. 2009;26(10):888-901.

25. Kleiman V, Clarke H, Katz J. Sensitivity to pain traumatization: a higher-order factor underlying pain-related anxiety, pain catastrophizing and anxiety sensitivity among patients scheduled for major surgery. Pain Res Manag. 2011;16(3):169-177.

26. Aldrich S, Eccleston C, Crombez G. Worrying about chronic pain: vigilance to threat and misdirected problem solving. Behav Res Ther. 2000;38(5):457-470.

27. McCracken LM, Eccleston C. A prospective study of acceptance of pain and patient functioning with chronic pain. Pain. 2005;118(1-2): $164-169$.

28. Merskey H, Bogduk N; Task Force on Taxonomy of the International Association for the Study of Pain. Classification of Chronic Pain: Descriptions of Chronic Pain Syndromes and Definitions of Pain Terms. Seattle, WA: IASP Press; 1994.

29. Schneider W, Eschman A, Zuccolotto A. E-Prime User's Guide. Pittsburgh, PA: Psychology Software Tools Inc.; 2002.

30. Asmundson GJ, Wright KD, Hadjistavropoulos HD. Hypervigilance and attentional fixedness in chronic musculoskeletal pain: consistency of findings across modified stroop and dot-probe tasks. J Pain. 2005;6(8):497-506.

31. Vago DR, Nakamura Y. Selective attentional bias towards pain-related threat in fibromyalgia: preliminary evidence for effects of mindfulness meditation training. Cogn Ther Res. 2011;35(6):581-594.

32. Keogh E, Ellery D, Hunt C, Hannent I. Selective attentional bias for pain-related stimuli amongst pain fearful individuals. Pain. 2001;91(1-2):91-100.

33. Asmundson GJ, Kuperos JL, Norton GR. Do patients with chronic pain selectively attend to pain-related information?: Preliminary evidence for the mediating role of fear. Pain. 1997;72(1-2):27-32.

34. Snider BS, Asmundson GJ, Wiese KC. Automatic and strategic processing of threat cues in patients with chronic pain: a modified stroop evaluation. Clin J Pain. 2000;16(2):144-154.

35. Melzack R. The McGill Pain Questionnaire: major properties and scoring methods. Pain. 1975;1(3):277-299.

36. Kučera H, Francis WN. Computational Analysis of Present-Day American English. Providence, RI: Brown University Press; 1967.

37. Roelofs J, Peters ML, Fassaert T, Vlaeyen JW. The role of fear of movement and injury in selective attentional processing in patients with chronic low back pain: a dot-probe evaluation. J Pain. 2005;6(5): 294-300.

38. Salvucci DD, Goldberg JH. Identifying fixations and saccades in eyetracking protocols: Proceedings of the Eye Tracking Research and Applications Symposium, Palm Beach Gardens, Florida, United States, 6-8 November 2000. New York, NY: ACM Press; 2000.

39. Komogortsev OV, Gobert DV, Jayarathna S, Koh DH, Gowda S. Standardization of automated analyses of oculomotor fixation and saccadic behaviors. IEEE Trans Biomed Eng. 2010;57(11):2635-2645.

40. Pollard CA. Preliminary validity study of the pain disability index. Percept Mot Skills. 1984;59(3):974.

41. Tait RC, Chibnall JT, Krause S. The Pain Disability Index: psychometric properties. Pain. 1990;40(2):171-182.

42. Sullivan MJL, Bishop SR, Pivik J. The pain catastrophizing scale: development and validation. Psychol Assess. 1995;7(4):524-532.

43. McCracken LM. "Attention" to pain in persons with chronic pain: a behavioral approach. Behav Ther. 1997;28(2):271-284.

44. Vowles KE, McCracken LM, McLeod C, Eccleston C. The Chronic Pain Acceptance Questionnaire: confirmatory factor analysis and identification of patient subgroups. Pain. 2008;140(2):284-291.

45. McCracken LM, Vowles KE, Eccleston C. Acceptance of chronic pain: component analysis and a revised assessment method. Pain. 2004;107(1-2):159-166. 
46. Spielberger CD, Gorsuch RL, Lushene RE, Vagg PR, Jacobs GA. Manual for the State-Trait Anxiety Inventory. Palo Alto, CA: Consulting Psychologists Press; 1983.

47. Spielberger CD. Anxiety: Current Trends in Theory and Research. New York, NY: Academic Press, Inc.; 1972.

48. Spielberger CD. State-Trait Anxiety Inventory: A Comprehensive Bibliography. Palo Alto, CA: Consulting Psychologists Press; 1989.

49. Taylor S, Zvolensky MJ, Cox BJ, et al. Robust dimensions of anxiety sensitivity: development and initial validation of the Anxiety Sensitivity Index-3. Psychol Assess. 2007;19(2):176-188.

50. Wheaton MG, Deacon BJ, McGrath PB, Berman NC, Abramowitz JS. Dimensions of anxiety sensitivity in the anxiety disorders: evaluation of the ASI-3. J Anxiety Disord. 2012;26(3):401-408.

51. Taylor S. The structure of fundamental fears. J Behav Ther Exp Psychiatry. 1993;24(4):289-299.

52. Bonferroni CE. Teoria Statistica delle Classi e Calcolo delle Probabilità. [Statistical class theory and calculation of probability]. Firenze, Italy: Libreria internazionale Seeber; 1936. Italian.

53. Heckman JJ. Sample selection bias as a specification error. Econometrica. 1979;47(1):153-161.

54. Rubin DB. Inference and missing data. Biometrika. 1976;63(3): 581-592.

55. Duchowski A. Eye Tracking Methodology: Theory and Practice. 2nd ed. London, UK: Springer-Verlag London Limited; 2007.

56. Cramer D. Basic Statistics for Social Research: Step-by-Step Calculations and Computer Techniques using Minitab. New York, NY: Routledge; 1997.

57. Huynh H, Feldt LS. Estimation of the Box correction for degrees of freedom from sample data in randomized block and split-plot designs. J Educ Behav Stat. 1976;1(1):69-82.

58. Schmukle SC. Unreliability of the dot probe task. Eur J Pers. 2005;19(7):595-605.

59. Dehghani M, Sharpe L, Nicholas MK. Selective attention to painrelated information in chronic musculoskeletal pain patients. Pain. 2003;105(1-2):37-46.

60. Khatibi A, Dehghani M, Sharpe L, Asmundson GJ, Pouretemad H. Selective attention towards painful faces among chronic pain patients: evidence from a modified version of the dot-probe. Pain. 2009;142(1-2): $42-47$
61. Pratto F, John OP. Automatic vigilance: the attention-grabbing power of negative social information. J Pers Soc Psychol. 1991;61(3): 380-391.

62. Ohman A. The role of the amygdala in human fear: automatic detection of threat. Psychoneuroendocrinology. 2005;30(10):953-958.

63. Öhman A, Wiens S. The concept of an evolved fear module and cognitive theories of anxiety. In: Manstead ASR, Frijda N, Fischer A, editors. Feelings and Emotions: The Amsterdam Symposium. Cambridge, UK: Cambridge University Press; 2004:58-80.

64. Sullivan MJ, Thorn B, Haythornthwaite JA, et al. Theoretical perspectives on the relation between catastrophizing and pain. Clin J Pain. 2001;17(1):52-64.

65. Roth RS, Geisser ME, Theisen-Goodvich M, Dixon PJ. Cognitive complaints are associated with depression, fatigue, female sex, and pain catastrophizing in patients with chronic pain. Arch Phys Med Rehabil. 2005;86(6):1147-1154.

66. McCracken LM, Iverson GL. Predicting complaints of impaired cognitive functioning in patients with chronic pain. J Pain Symptom Manage. 2001;21(5):392-396.

67. Vlaeyen JW, Linton SJ. Fear-avoidance and its consequences in chronic musculoskeletal pain: a state of the art. Pain. 2000;85(3):317-332.

68. Eccleston C, Crombez G. Worry and chronic pain: a misdirected problem solving model. Pain. 2007;132(3):233-236

69. McCracken LM, Eccleston C. Coping or acceptance: what to do about chronic pain? Pain. 2003;105(1-2):197-204.

70. Viane I, Crombez G, Eccleston C, Devulder J, De Corte W. Acceptance of the unpleasant reality of chronic pain: effects upon attention to pain and engagement with daily activities. Pain. 2004;112(3):282-288.

71. Bar-Haim Y, Lamy D, Pergamin L, Bakermans-Kranenburg MJ, van IJzendoorn MH. Threat-related attentional bias in anxious and nonanxious individuals: a meta-analytic study. Psychol Bull. 2007;133(1):1-24.

72. Crombez G, Eccleston C, Van Damme S, Vlaeyen JW, Karoly P. Fearavoidance model of chronic pain: the next generation. Clin J Pain. 2012;28(6):475-483
Journal of Pain Research

\section{Publish your work in this journal}

The Journal of Pain Research is an international, peer-reviewed, open access, online journal that welcomes laboratory and clinical findings in the fields of pain research and the prevention and management of pain. Original research, reviews, symposium reports, hypothesis formation and commentaries are all considered for publication.

\section{Dovepress}

The manuscript management system is completely online and includes a very quick and fair peer-review system, which is all easy to use. Visit http://www.dovepress.com/testimonials.php to read real quotes from published authors. 